\title{
Etude de l'injection de charge sur des plaques d'élastomère silicone par mesures du potentiel de surface
}

\author{
Philippe Molinié* — Daniel Alvarez** \\ *Supélec, Département Energie \\ Plateau du Moulon, 91192 Gif-sur-Yvette Cedex. \\ philippe.molinie@supelec.fr \\ ** Gumelast, Santovenia de Pisuerga \\ 47155 Valladolid, Espagne \\ dani.alvarez@gumelast.com
}

\begin{abstract}
RÉSUMÉ. Nous présentons ici des mesures de potentiel de surface sur des plaques d'élastomères silicones vulcanisées à chaud, qui démontrent l'existence pour des champs de l'ordre de $1 \mathrm{kV} / \mathrm{mm}$ de phénomènes d'injection de charge importants, et d'un transport rapide de la charge dans l'isolant, témoignant de l'absence de piégeage profond dans le matériau. Certaines observations suggèrent également que la charge électrique semble pouvoir migrer dans le matériau dans une direction opposée au champ. Ces résultats diffèrent nettement de ce qui est généralement observé dans les polymères utilisés en isolation, et méritent des investigations supplémentaires, car pour l'instant, les mécanismes de transport et d'injection de charge dans les élastomères silicones, contrairement à leurs propriétés de surface, ont fait l'objet de fort peu d'études.
\end{abstract}

ABSTRACT. Surface potential measurements have been performed on high temperature vulcanized silicon rubber plates, showing the evidence of important injection phenomena, occurring for fields about $1 \mathrm{kV} / \mathrm{mm}$, together with fast charge transport phenomena into the insulator, implying the lack of deep traps in the material. Some results also suggest that the electrical charge may drift into the material against the field. These results are quite different from what is usually observed on insulating polymers, and deserve extra investigation, since up to now, charge transport and injection mechanisms in silicone rubber have been scarcely studied, in contrast with its surface properties.

MOTS-CLÉS : caractérisation, isolation, potentiel de surface, triboélectricité, injection de charges, élastomères silicones, isolateurs.

KEYWORDS: characterization, insulation, surface potential, triboelectricity, charge injection, silicone rubber, insulators 


\section{Introduction}

Les études sur la charge d'espace se sont multipliées depuis deux décennies dans le domaine de l'isolation polymérique haute tension. Elles ont permis par exemple une plus grande maîtrise des phénomènes initiateurs du vieillissement des câbles, des condensateurs, des transformateurs, ainsi que le développement de nouveaux matériaux. Les phénomènes d'injection et de transport de la charge ont donc été largement étudiés pour divers matériaux utilisés dans ces installations, à commencer par le polyéthylène et le polypropylène. En revanche, les mécanismes de transport et d'injection de charge dans les élastomères silicones, qui sont pourtant de plus en plus utilisés en isolation, que ce soit pour le gainage de câbles ou pour les isolateurs composites, ont fait l'objet de fort peu d'études. Ce sont les propriétés de surface, notamment le recouvrement de l'hydrophobie, qui ont fait l'objet de la préoccupation industrielle la plus forte, et ont été les plus étudiées (Cherney 1999, Moreno et al. 2001, Yoshimura et al. 1999) et les études sur les propriétés électriques volumiques se sont souvent limitées à la spectroscopie diélectrique (Nguyen et al. 2004, Tuncer et al. 2000). L'étude de l'injection et du transport de la charge dans ces matériaux est donc un domaine de recherche relativement inexploré, et pourtant les résultats que nous présentons font apparaître pour certaines formulations un comportement extrêmement original, très différent des polyoléfines.

\section{Expériences}

Les expériences ont été menées sur des plaques d'élastomère silicone vulcanisé à chaud (EVC), fortement chargés en alumine, d'épaisseur $2 \mathrm{~mm}$ et d'environ $120 \mathrm{~mm}$ de côté, placées sur un porte-échantillon de laiton. Plusieurs formulations ont été étudiées, les résultats obtenus étant qualitativement proches. Par souci de clarté nous présenterons ici les résultats obtenus sur une seule de ces formulations. Certaines plaques ont été métallisées sur leur surface inférieure en utilisant une peinture au noir de carbone ; si ce n'est pas spécifié, leurs deux surfaces sont libres.

Nous utilisons un banc de mesure, comprenant un poste de charge par décharge couronne, et un poste de mesure de potentiel. La plaque sur son porte-échantillon est déplacée par un vérin entre les deux postes. Pendant la charge, le potentiel de surface est régulé par une grille entre la source corona et l'échantillon, selon le schéma classique. Nous utilisons deux postes de charge au choix : un dispositif de charge « local » de type pointe-grille circulaire, permettant de déposer un disque de charge sur la surface, d'un diamètre $(17 \mathrm{~mm})$ nettement inférieur aux dimensions de l'échantillon, et un dispositif linéique fil-grille qui permet, par balayage, de charger la surface totale de la plaque. Avec le premier dispositif, le temps de charge est d'une seconde ; avec le deuxième, il est de l'ordre de 10 secondes pour l'ensemble de la surface. Les courants de charge sont de l'ordre de 10 à $20 \mu \mathrm{A}$.

La mesure du potentiel de surface est réalisée par un voltmètre électrostatique équipé d'une sonde classique Monroe, à condensateur vibrant, asservie au potentiel 
de la surface. La résolution latérale de la sonde est de l'ordre de 0,5 $\mathrm{mm}$. Cette sonde est mobile sur un axe, le porte-échantillon étant mobile sur l'axe perpendiculaire, ce qui permet une cartographie en deux dimensions du potentiel (dont la durée est de l'ordre d'une heure - les cartographies présentées étant par ailleurs généralement réalisées après 1 heure d'attente).

Nous avons effectué de nombreuses séries de mesures, alternant après une charge initiale l'enregistrement du déclin de potentiel en mode «ponctuel» (voir figure 1) et des cartographies successives pour divers niveaux de charge. Le champ maximum appliqué est faible, inférieur dans tous les cas à $1 \mathrm{kV} / \mathrm{mm}$. Enfin, nous avons systématiquement neutralisé la surface après les mesures, et effectué des cartographies ou un suivi du « retour de potentiel » après neutralisation. Les mesures ont été réalisées à une température de $20^{\circ} \mathrm{C}$ et une humidité relative d'environ $50 \%$.

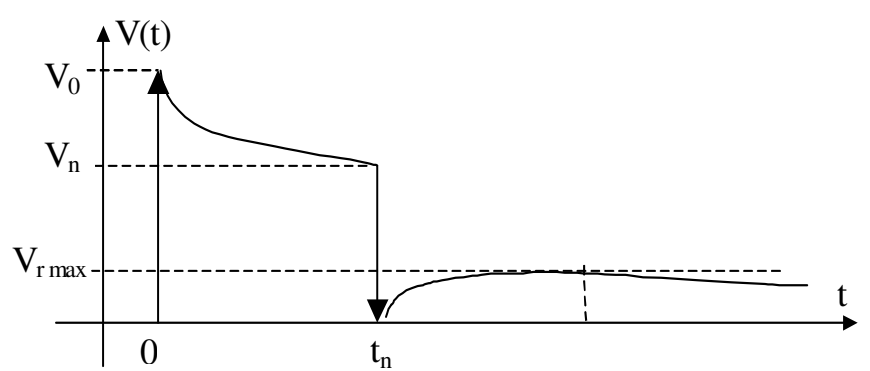

Figure 1. Déclin et retour de potentiel (mode « ponctuel»)

\section{Résultats}

\subsection{Effets triboélectriques}

La manipulation des plaques nous a permis de constater que des potentiels importants apparaissaient dès qu'on les déplaçait de leur porte échantillon métallique. Plusieurs séries de mesures ont permis d'évaluer ces effets triboélectriques.

\subsubsection{Mesures du potentiel après retournement (face supérieure / inférieure)}

Le simple retournement d'une plaque laissée au repos pendant plusieurs jours et soigneusement neutralisée, à plusieurs reprises, fait apparaître sur la surface des potentiels élevés : la cartographie présentée figure 2 a montre des valeurs qui peuvent atteindre $1500 \mathrm{~V}$. La distribution du potentiel fait apparaître de nombreuses petites tâches circulaires ou ovales, positives ou négatives, de quelques millimètres de diamètre, dont le potentiel diffère de plus de 1000 V de leur entourage. 


\subsubsection{Mesures après rotation}

Le fait de décoller lentement une plaque, de la tourner d'un quart de tour et de la repositionner, sans retournement, produit également des zones à potentiels élevés et des tâches: dans le cas d'une plaque initialement neutre, ces potentiels sont symétriques (figure 2b). Dans le cas d'une plaque préalablement chargée positivement, par contre, on remarque l'apparition de tâches négatives ; dans le cas d'une plaque chargée négativement, de tâches à potentiel nul. Nous n'avons pas pu mesurer précisément le temps d'apparition de ces zones à fort potentiel, ou de ces tâches à polarité inversée, mais il est inférieur à cinq minutes.

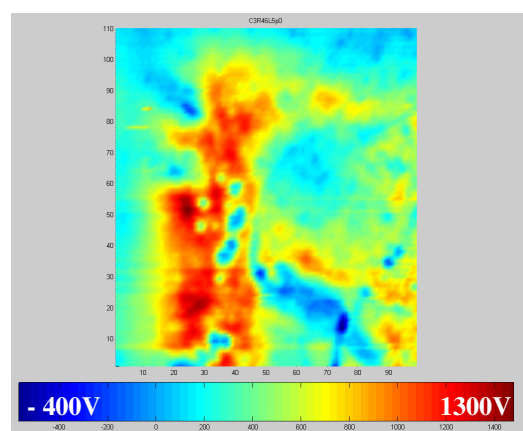

(a)

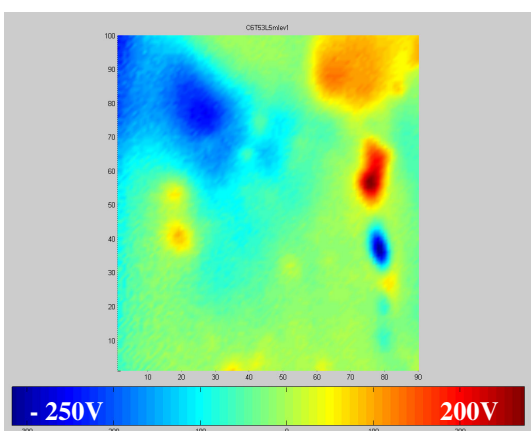

(b)

Figure 2. Cartographie (100x100mm) de potentiel après retournement (a) ou: décollement, rotation et repositionnement $(b)$, d'une plaque neutre

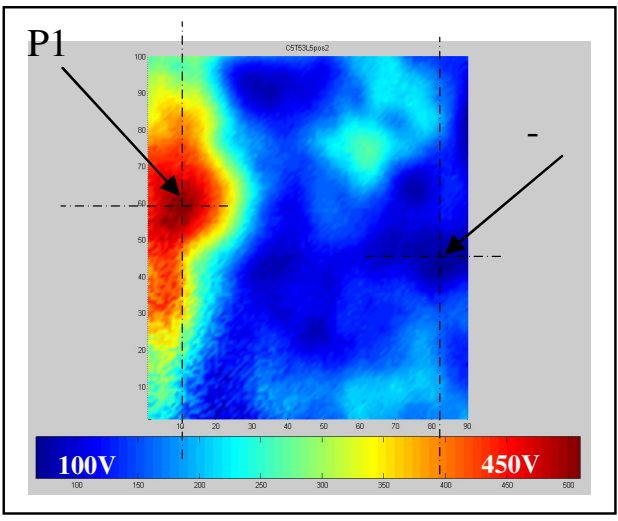

Figure 3. Cartographie $(100 \mathrm{~mm} \times 100 \mathrm{~mm})$ de potentiel après charge à $+2000 \mathrm{~V}$ et de $60 m n$ d'attente (bas de la cartographie) à 120mn(haut). 


\subsection{Cartographies : déclins après charge}

\subsubsection{Hétérogénéité et dépendance de la polarité de la charge déposée}

Les cartographies de potentiel montrent une grande hétérogénéité de comportement suivant le point considéré (figure 3). Quelque soit la formulation du matériau, cette hétérogénéité se retrouvait toujours présente, mais de manière plus ou moins marquée suivant les cas, et dans des zones disposées différemment. Elle a donc d'abord été attribuée à une hétérogénéité de composition, ce qui a été remis en cause par la suite. Sur chaque plaque, nous avons défini des zones bien caractéristiques à comportement opposé («P1» à faible déclin de potentiel, et «P2 » à fort déclin), pour les étudier ensuite en mode "ponctuel".

L'observation de cartographies réalisées en polarité négative et positive montre que le potentiel est de $15 \%$ à $70 \%$ plus faible en polarité négative après $90 \mathrm{mn}$ de déclin, pour des valeurs de charge déposée identiques en valeur absolue. La répartition des zones semble cependant dépendre peu du signe de la charge déposée.

\subsubsection{Dépendance du contact métal - plaque}

Nous avons trouvé que les zones observées étaient essentiellement liées au contact entre la plaque et le porte échantillon en laiton. Dans la plupart des cas, la rotation de la plaque d'un quart de tour ne se traduit pas par une rotation de la plupart des zones observées. Après avoir exclu la possibilité d'une hétérogénéité du dépôt de charge lui-même, nous avons conclu à l'influence prépondérante du contact avec le porte échantillon, dépendant, par exemple, de l'oxydation de celui-ci. Pour vérifier cette influence du contact à la contre-électrode, une série de plaques a été métallisée par une peinture au noir de carbone, en laissant libre une zone en forme caractéristique de "V".

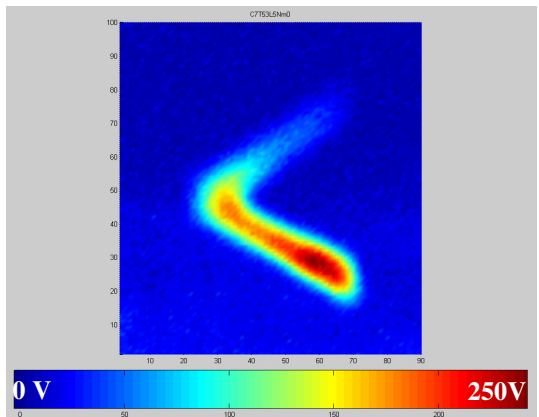

(a) plaque neutre

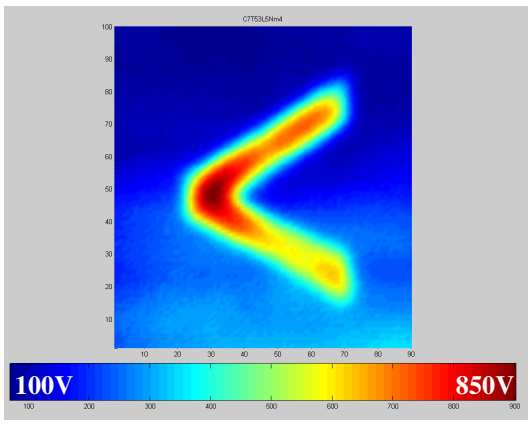

(b) $3^{\text {ème }}$ charge successive

Figure 4. Cartographie (100mmx100mm) après métallisation évidée en «V», après charge à $+2000 \mathrm{~V}$ et de $30 \mathrm{mn}$ d'attente (bas de la cartographie) à 90mn(haut). 
Les résultats ont été nets, le potentiel chutant brutalement partout $(50 \mathrm{~V}$ après $30 \mathrm{mn}$, contre typiquement $300 \mathrm{~V}$ dans les zones «P2 » et $700 \mathrm{~V}$ dans les zones «P1 »), sauf dans la zone dont l'arrière n'était pas métallisé (figure 4a). Nous avons remarqué également que des charges successives dans la même polarité conduisent à une augmentation des potentiels, (figure $4 b$ ).

\subsection{Déclin et retour de potentiel}

\subsubsection{Enregistrement du déclin de potentiel aux points caractéristiques}

Le déclin et le retour de potentiel ont été enregistrés en mode «ponctuel », pour chaque plaque, aux points P1 et P2 donnés par la figure 3.

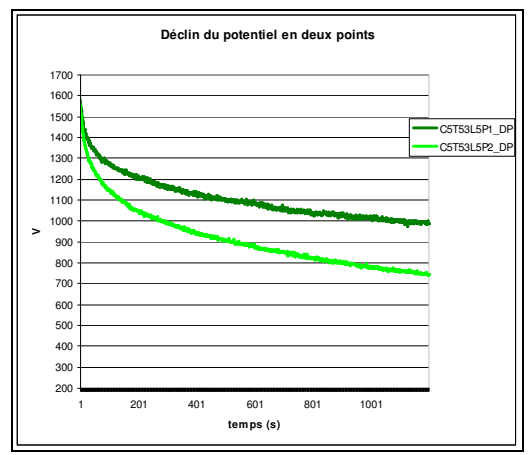

Figure 5. Déclin de potentiel aux points «P1» et «P2»

Deux traitements de ces données sont présentés, suivant les principes que nous avons présenté dans une étude précédente (Llovera et al., 2004). La dérivée, en coordonnées bilogarithmiques, présente l'allure classique d'une courbe en $t^{-n}$ (figure 6a). La transformée $t d V / d t$ ou $d V / d \log t$ en fonction de logt est un outil utile pour visualiser les temps caractéristiques du phénomène, sans préjuger de ses causes physiques. Elle est représentée, normalisée au potentiel initial $\mathrm{V}_{0}$, sur la figure $6 \mathrm{~b}$.

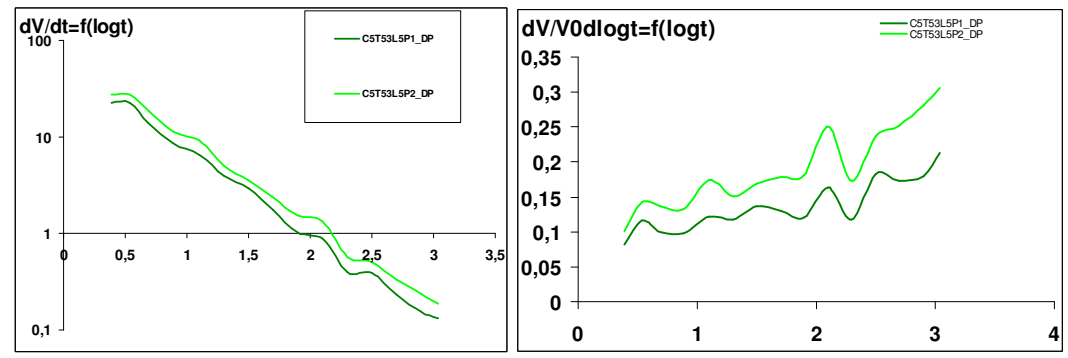

Figure 6. Traitements de la courbe de déclin de potentiel 


\subsubsection{Enregistrement du retour et comparaison}

Nous avons procédé à une neutralisation rapide immédiatement après l'enregistrement des courbes de déclin précédentes. Les courbes de retour de potentiel après neutralisation sont données figure 7a. Les valeurs de retour de potentiel sont supérieures à $30 \%$ de la tension au moment de la neutralisation.

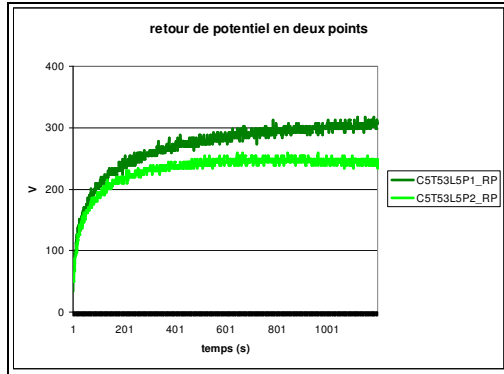

(a)

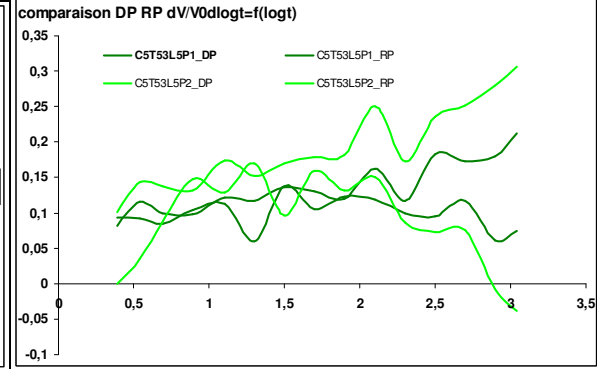

(b)

Figure 7. Retour de potentiel et traitement

Il est intéressant de comparer, sur la représentation $d V / d \log t=f(\log t)$, les représentations du déclin et du retour, normalisés, dans le cas du déclin, à la tension de charge, et dans celui du retour, à la tension de neutralisation (figure $7 b$ ). Nous voyons que les courbes de déclin et de retour, pour le point $\mathrm{P} 1$ comme pour le point $\mathrm{P} 2$, sont proches pour les temps courts, l'écart se creusant ensuite. Ces résultats, comme les précédents, se retrouvent pour les différentes formulations testées.

\section{Modèles de déclin et de retour de potentiel}

\subsection{Introduction}

Nous allons présenter ici de manière succinte les principaux modèles du déclin et du retour de potentiel, pour interpréter les résultats de l'expérience décrite en figure 1. L'échantillon est chargé à un instant initial à un potentiel $V_{0}$, le déclin de potentiel est alors enregistré jusqu'à la tension $V_{n}$ à l'instant $t_{n}$, où il est neutralisé. On enregistre ensuite le retour de potentiel. Nous considérons notre isolant comme une plaque de permittivité $\varepsilon$, dont les dimensions latérales sont très grandes par rapport à son épaisseur L, et donc on suppose que les grandeurs physiques (champ, potentiel, densité de charge) ne dépendent que de l'éloignement à la masse.

Les principaux mécanismes pouvant intervenir pour expliquer la dynamique du déclin et du retour de potentiel sont l'injection de charge, la conduction, et la polarisation dipolaire (Molinié, 2005). 


\subsection{Injection de charge}

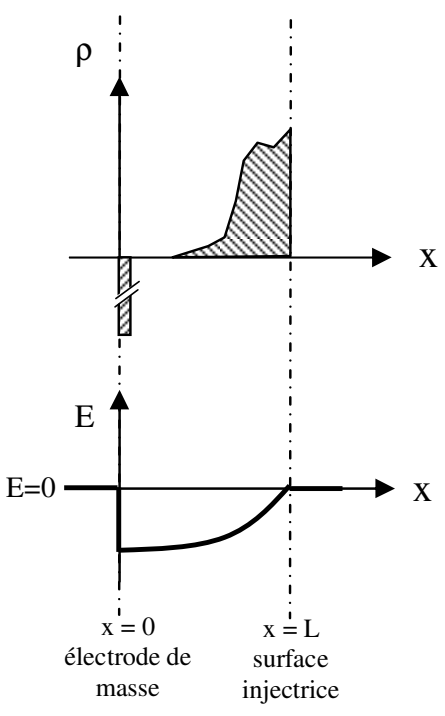

a) avant neutralisation

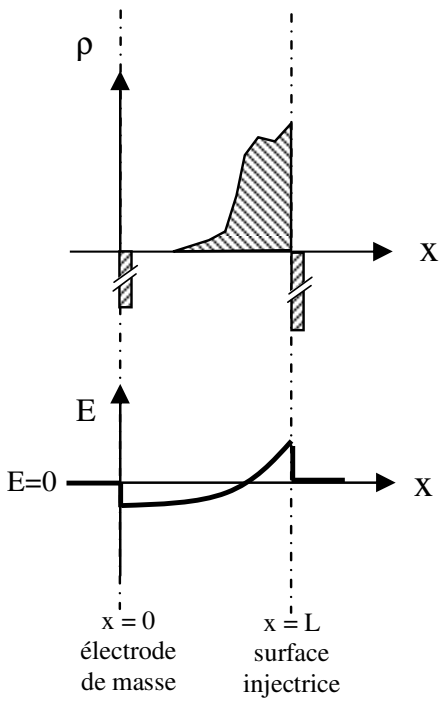

b) après neutralisation

Figure 8. Densité de charge et champ dans l'isolant avant et après neutralisation

\subsubsection{Dynamique du déclin de potentiel}

Supposons qu'à un instant donné, une charge d'espace ait été injectée dans l'isolant, avec une distribution $\rho(\mathrm{x})$, comme le décrit la figure 8 . On peut aisément montrer que :

$$
V(d)=\frac{1}{\varepsilon} \int_{0}^{L} x \rho d x
$$

Ce qui donne, en introduisant la profondeur moyenne d'injection des charges $<\lambda>=\int_{0}^{L}(d-x) \rho d x / \int_{0}^{L} \rho d x$ (en incluant les charges qui ont rejoint la masse, comme étant en $\mathrm{x}=0$ ), et le potentiel $\mathrm{V}_{0}$ avant toute injection, la relation suivante, qui est tout à fait générale :

$$
V(L)=V_{0}\left(1-\frac{<\lambda>}{L}\right)
$$


Le déclin de potentiel, exprimé par $d V / V_{0} d t$, est donc dans les modèles d'injection proportionnel à la vitesse de pénétration des charges dans l'échantillon (exprimée en termes relatifs à l'épaisseur, par $\frac{1}{L} \frac{d<\lambda>}{d t}$ ).

\subsubsection{Retour de potentiel}

Lors de la neutralisation de l'échantillon, la profondeur moyenne de la charge injectée étant posée égale à $\left\langle\lambda_{n}\right\rangle$, on va déposer sur la surface une charge de signe opposé, dont la densité sera $\sigma_{n}=-\frac{\varepsilon V_{0}}{L}\left(1-\frac{<\lambda_{n}>}{L}\right)$. Mais on peut alors remarquer que le champ dans l'isolant, au voisinage de la surface, valant $-\sigma_{n} / \varepsilon$, a changé de signe. Comme le montre également la figure 8 , on voit qu'il existe alors dans l'isolant un plan de champ nul. La partie de la distribution interne de charge la plus proche de la surface va alors retourner vers celle-ci, attirée par la charge de neutralisation, pendant que la charge située de l'autre côté du plan de champ nul poursuivra son mouvement d'injection. Au bout d'un temps infini, la charge totale étant évacuée de l'isolant, le champ sera nul dans l'ensemble de celui-ci, et le potentiel tendra vers 0 . La dynamique du phénomène transitoire dépendra des quantités relatives de charge de part et d'autre du plan de champ nul. La densité de charge contribuant au retour sera, d'après le théorème de Gauss, l'opposé de la charge de neutralisation, soit $\sigma_{r}=\frac{\varepsilon V_{0}}{L}\left(1-\frac{<\lambda_{n}>}{L}\right)$, alors que la densité de charge contribuant à la poursuite de l'injection sera $\sigma_{d}=\sigma_{0}-\sigma_{n}-\sigma_{g}=\frac{\varepsilon V_{0}<\lambda_{n}>}{L^{2}}-\sigma_{g}$ ( $\sigma_{g}$ étant la charge qui a atteint la masse). Les champs crées par ces charges sont proportionnelles à ces densités, donc on peut en conclure que leurs vitesses de déplacement seront proportionnelles au carré de ces densités. Deux cas peuvent alors se présenter : si la distribution de la charge au moment de la neutralisation est majoritairement plus proche de la surface injectrice que de la contre-électrode, il y aura un retour de potentiel de même signe que le potentiel initial, le mouvement des charges revenant vers l'électrode injectrice étant plus massif et plus rapide que le mouvement des charges continuant à s'injecter. Dans le cas où la distribution serait au contraire plus proche de la contre électrode, il faut envisager au contraire un retour de potentiel "anormal", de signe opposé au potentiel initial.

\subsubsection{Rôle du piégeage}

Des modèles de déclin de potentiel ont été développés dans différents cas de figure (voir Molinié 2005). En l'absence de phénomènes de piégeage, un modèle simple prévoit une injection par plans de charge gardant une vitesse constante pendant leur traversée de l'échantillon. La vitesse de décroissance du potentiel 
devrait alors être constante tant que les premières charges injectées n'ont pas atteint la contre électrode.

Dans la pratique, on observe rarement cela, les phénomènes de piégeage et dépiégeage des charges jouant un rôle essentiel dans la dynamique du déclin. D'une part, il est fréquent que les charges soient initialement piégées à divers niveaux d'énergie en surface, de telle sorte que l'émission progressive des charges de surface puisse être le facteur limitant du déclin, le transport étant ensuite relativement facile. D'autre part, le désordre et l'existence de pièges dans le matériau vont rendre le transport dispersif, et conduire à des lois de déclin de potentiel qui dépendront de la répartition énergétique du piégeage. Concernant le retour de potentiel, il faut ajouter qu'à notre connaissance, il n'a pas été possible à ce jour d'attribuer de manière convaincante des résultats à l'influence prépondérante de l'injection de charge.

\subsection{Conduction intrinsèque}

L'existence d'une conduction intrinsèque doit en principe conduire à une forme exponentielle pour le déclin de potentiel. Comme elle se traduit par une neutralisation progressive de la charge de surface, sans accumulation de charge interne (le cas de la polarisation interfaciale rentre dans le paragraphe suivant), elle ne peut pas donner naissance à un retour de potentiel.

\subsection{Polarisation dipolaire : modèle de relaxation linéaire}

\subsubsection{Cas général}

La plupart des isolants présentent une composante lente de la polarisation dipolaire, qui peut être liée, par exemple, pour les polymères, à des réorientations moléculaires du matériau sous l'influence du champ électrique. La description générale de ces phénomènes peut se faire en les supposant linéaires en fonction du champ. Ils entrent alors dans la catégorie plus générale des phénomènes de relaxation : l'isolant peut être décrit par une fonction dite d'absorption $\phi(t)$. La relation entre le potentiel et la charge déposée sur l'isolant, de $V(t)=\frac{L q(t)}{\varepsilon}$, devient, pour prendre en compte les effets retardés de la permittivité (voir Molinié, 2005) :

$$
V(t)=\frac{L}{\varepsilon} \int_{-\infty}^{t} q(\tau) \phi_{E}(t-\tau) d \tau
$$

( $\varepsilon$ étant la permittivité statique, incluant tous les phénomènes de polarisation jusqu'à la première mesure de potentiel ; $\phi_{E}$ intègre les phénomènes de polarisation plus lents) 
Si on dépose sur l'isolant une charge $q_{0}$ à l'instant $t=0$, on a : $q(t)=\Gamma_{0}(t) q_{0}$, $\Gamma_{0}(t)$ étant un créneau (fonction de Heavyside) centré en $\mathrm{t}=0$, et donc, en prenant la dérivée de l'équation précédente :

$$
\frac{d V(t)}{d t}=\frac{L q_{0}}{\varepsilon} \frac{d}{d t} \int_{0}^{t} \phi_{E}(\theta) d \theta=\frac{L q_{0}}{\varepsilon} \phi_{E}(t)
$$

La dérivée du potentiel est proportionnelle à la fonction d'absorption $\phi_{E}(t)$.

Si on neutralise l'isolant à l'instant $t_{n}$, en déposant une charge $q_{n}$ de signe opposé à $q_{0}$, l'évolution du potentiel après la neutralisation est donnée, en régime linéaire, par la superposition du signal lié au dépôt initial et de celui induit par le créneau de neutralisation :

$$
\frac{d V(t)}{d t}=\frac{L q_{0}}{\varepsilon} \phi_{E}(t)+\frac{L q_{n}}{\varepsilon} \phi_{E}\left(t-t_{n}\right)
$$

$\phi_{E}(t)$ étant une fonction (en valeur absolue) décroissante du temps, le deuxième terme, de signe opposé au premier, l'emportera généralement pour les petites valeurs de $t$ - $t_{n}$, entraînant une évolution de $d V / d t$ opposée à l'évolution initiale, donc un retour de potentiel. Puis, au bout d'un temps suffisamment long, le premier terme l'emportera à nouveau, la valeur de $q_{0}$ étant plus élevée, en valeur absolue, que celle de $q_{n}$.

4.4.2. Cas spécifique (fréquent) dans lequel la fonction $\phi_{E}(t)$ est en loi de puissance

Souvent dans les solides isolants, nous trouvons, dans un grand intervalle de temps, vérifiée la relation :

$$
\left|\frac{\phi_{E}(t)}{\phi_{E}\left(t_{0}\right)}\right|=\left(\frac{t}{t_{0}}\right)^{-n} \quad \mathrm{n} \text { étant compris entre } 0,5 \text { et } 1
$$

La décroissance rapide de $\phi_{E}(t)$ entraîne, pour un temps court après la neutralisation :

$$
\frac{d V(t)}{d t}=\frac{L q_{0}}{\varepsilon} \phi_{E}(t)+\frac{L q_{n}}{\varepsilon} \phi_{E}\left(t-t_{n}\right) \approx \frac{L q_{n}}{\varepsilon} \phi_{E}\left(t-t_{n}\right) \approx V_{n} \phi_{E}\left(t-t_{n}\right)
$$

Comme le déclin de potentiel initial s'exprimait par $\frac{d V(t)}{d t}=\frac{L q_{0}}{\varepsilon} \phi_{E}(t)=V_{0} \phi_{E}(t)$, on en déduit que, pour les temps courts après la neutralisation :

$$
\frac{d V(t)}{V_{n} d t} \approx \frac{d V\left(t-t_{n}\right)}{V_{0} d t}
$$


On peut calculer également dans le cadre du modèle linéaire le temps où le retour de potentiel atteint son maximum. Il est obtenu pour $V_{0} \phi_{E}(t)+V_{n} \phi_{E}\left(t-t_{n}\right)=0$

$$
\begin{gathered}
\text { c'est-à-dire pour } \frac{V_{0}}{V_{n}}=-\left(\frac{t-t_{n}}{t}\right)^{-n} \text { c'est à dire pour : } \\
t-t_{n}=t_{n} \frac{1}{\left(-\frac{V_{0}}{V_{n}}\right)^{\frac{1}{n}}-1}
\end{gathered}
$$

\section{Discussion}

\subsection{Transfert et injection de charge à l'interface métal silicone}

\subsubsection{Ampleur du transfert de charge}

Les résultats présentés dans la section 3.1.1 prouvent l'importance du transfert de charge qui se produit lors d'un simple contact du silicone avec le métal. Cette charge semble, sur une plaque bien neutralisée, se faire de manière relativement symétrique et par «points chauds » (figure 2a) : effet d'une décharge au moment du décollement?

Lorsque la plaque est chargée au moment du décollement, il semble que cela influe le transfert de charge, et que celui-ci s'effectue plus fortement lorsque la plaque est chargée positivement.

\subsubsection{Comportement d'injection très dépendant des caractéristiques de l'interface}

Nos expériences ont montré le rôle prédominant des effets de surface dans les hétérogénéité de cartographies observées. Elles semblent être liées au contact métalsilicone, essentiellement, et peu aux phénomènes intervenant du côté de la surface chargée par la décharge corona. Les mesures avec métallisation de la face inférieure confirment cet effet. Les résultats, présentés figure 4 montrent une spectaculaire injection de charge à la contre électrode, de manière surprenante car les champs sont faibles.

\subsection{Transport de la charge dans le volume}

\subsubsection{Existence d'un transport sans champ externe appliqué}

Les résultats de triboélectricité présentés sur la figure $2 \mathrm{~b}$ ne peuvent pas s'expliquer uniquement en termes de transfert de charge, car il faut faire intervenir également un transport de la charge suffisant pour expliquer la formation d'un potentiel visible sur la face opposée. La charge, doit, au minimum, surmonter 
l'attraction de sa charge image sur la contre-électrode de masse, pour conduire à un tel potentiel, c'est-à-dire qu'elle doit pouvoir se déplacer contre le champ électrique.

La distance de migration n'est pas connue, il est possible qu'elle soit limitée, par exemple à quelques dizaines de $\mu \mathrm{m}$, si une très grande densité de charges est injectée. Il est possible qu'il y ait un lien entre ce phénomène et les restructurations impliquées dans le recouvrement de l'hydrophobicité (voir plus loin).

\subsubsection{Temps de transit court}

Les résultats de la figure 4 montrent la grande mobilité d'une partie de la charge au moins, une fois injectée, et l'absence de repiégeage stable de cette charge lors de sa traversée du matériau.

On peut déduire de ces mesures une borne supérieure du temps de transit $\tau$ à travers la plaque, qui est de quelques minutes, ce qui permet d'estimer une mobilité $\mu=L^{2} / V \tau$ ( $L$ épaisseur, $V$ potentiel initial) de la charge supérieure à $10^{-7} \mathrm{~cm}^{2} \mathrm{~V}^{-1} \mathrm{~s}^{-1}$.

\subsection{Interprétation des déclins et retours de potentiel}

L'allure des courbes de potentiel ne permet pas, à elle seule, de préjuger du mécanisme physique impliqué : en effet, la loi observée (on a dans les deux cas une loi de puissance décroissante pour $\mathrm{dV} / \mathrm{dt}$ ) peut s'interpréter à la fois dans un cadre de polarisation dipolaire, par un phénomène de transport dispersif, ou par un dépiégeage progressif de la charge de surface (Molinié 2005).

Le résultat obtenu par la métallisation de la face inférieure permet cependant d'affirmer que ce ne sont pas les processus de transport dans le volume qui limitent le déclin de potentiel dans les deux cas «P1» et «P2»présentés ci-dessus. La quantité de charge présente dans le volume de l'échantillon après $20 \mathrm{mn}$ de déclin de potentiel ne devrait pas représenter plus de quelques dizaines de volts, du même ordre (voire inférieure) à ce qu'elle est dans le cas «métallisé ». L'injection de charge est limitée dans ce cas par les surfaces qui retiennent l'essentiel de la charge.

Quelle est alors l'importance relative des phénomènes de polarisation dipolaire et d'injection de charge? Une réponse peut être proposée en comparant les dynamiques des déclins et retour de potentiel.

D'après le modèle, dans le cas d'un phénomène de relaxation, les courbes correspondant au retour (normalisé à $V_{n}$ ) et au déclin (normalisé à $V_{0}$ ), dans la représentation de la figure $7 \mathrm{~b}$ devraient se confondre aux temps faibles (voir équation [8]). Dans le cas «P1 », les courbes sont très proches, tant que $t$-tn<t/10, en revanche elles sont plus éloignées dans le cas «P2 ».

Par ailleurs, $n$ peut être calculé à partir de la figure 6 a et vaut 0,88 pour le cas «P1 » et 0,86 pour le cas « $\mathrm{P} 2$ ». On peut en déduire d'après l'équation [9] la valeur théorique du temps après la neutralisation où le retour passera par un maximum dans le modèle de relaxation. On trouve: $\log t_{\max }=3,23$ dans le cas P1 
(expérimentalement : supérieure à 3,3, de l'ordre de 3,5 ?), et $\log t_{\max }=2,97$ dans le cas P2 (valeur mesurée 2,9).

On voit que le modèle de relaxation s'applique très bien aux mesures faites en P1. Dans le cas P2, il explique bien le retour, mais le fait que le déclin soit plus important conduit logiquement à proposer l'hypothèse d'une injection limitée par l'électrode qui se superpose (de manière minoritaire) au signal de déclin de potentiel lié à la polarisation, et qui apparaît peu (sauf peut-être dans les temps courts) sur le signal de retour de potentiel, étant donné la rapidité du transit dans le volume.

L'interprétation des cartographies du type présenté sur la figure 3 sera alors la suivante: les points au potentiel le plus élevé correspondent à des zones où l'injection se fait difficilement à la contre électrode, et où la valeur du potentiel est essentiellement déterminée par des phénomènes de relaxation; les points au potentiel les plus bas correspondent à des points où les deux phénomènes se superposent. Ces zones sont dépendantes, à la fois de l'état de surface de la plaque, et de celui du métal, mais, même pour les points à faible potentiel, le déclin de potentiel est limité par l'émission de l'électrode plus que par le temps de transit dans le matériau.

Dans le cas d'une contre-électrode peinte, il est possible (mais pas certain) que ce soit le temps de transit dans le matériau qui limite le déclin.

\section{Conclusions}

Les résultats les plus inattendus pour nous de ce travail sont, d'une part, la démonstration de la grande facilité avec laquelle, à champ très faible, on peut injecter à partir d'une électrode métallique des charges dans des élastomères silicones, charges qui seront ensuite très mobiles dans le matériau, et d'autre part, la mise en évidence de phénomènes de transport de la charge lors des essais de triboélectricité qui ne sont pas liés au champ électrique.

Il est possible que ces deux observations soient liées à l'existence dans ce type de matériaux de mécanismes qui assurent le recouvrement d'hydrophobicité du matériau et justifient son emploi comme revêtement des isolateurs. En effet, une altération chimique de la surface du matériau sera rapidement compensée par des restructurations internes, faisant intervenir notamment la migration d'oligomères à faible poids moléculaire, de telle sorte que les fonctions polaires sont éloignées de la surface. Ces phénomènes sont bien connus, et ont été maintes fois étudiés (Cherney 1999, Yoshimura et al. 1999, Moreno et al. 2001). Des phénomènes comparables peuvent-ils intervenir lors d'une simple charge du matériau, à la suite du contact avec un métal ou à la suite d'un dépôt d'ions par la décharge couronne ? Nous manquons d'information sur cette question, mais ce type d'hypothèse semble plausible, notamment pour expliquer un transport de la charge apparemment contre le champ électrique appliqué. La présence par ailleurs de phénomènes de relaxation 
dipolaire n'est pas surprenante, étant donné le caractère polaire des chaînes d'élastomères silicones.

La principale limite de cette étude, est bien entendu l'utilisation d'une seule technique de mesures, et l'utilisation de techniques de mesures de charge d'espace apporterait certainement des résultats complémentaires. Par ailleurs, on ne sait pas si ces résultats, à notre connaissance nouveaux, et que nous avons obtenus pour quelques formulations bien particulières du matériau, traduisent un comportement général sur les silicones, ou si, par exemple, ils sont liés à la forte densité de charges minérales présente dans nos échantillons.

Quoiqu'il en soit, la mise en évidence de ces propriétés bien particulières de certains types d'élastomères silicones EVC peut permettre d'espérer des applications. Par exemple, cette injection massive et ce transit relativement facile de la charge à champ faible, peut en faire un matériau «modèle » pour des études théoriques sur l'injection et le transport de charge; on peut également penser que l'utilisation de ce type de plaque peut permettre de visualiser de manière extrêmement nette des altérations de surface sur des métaux. Enfin, il est probable que ces effets d'injection de charge à champ faible dans les élastomères silicone sont insuffisamment pris en compte dans la mise en oeuvre des systèmes d'isolation qui les utilisent, et l'amélioration de leur fiabilité ne pourra que profiter de ces résultats.

\section{Références}

Cherney E.A, Gorur R.S., «RTV Silicone Rubber Coatings for Outdoor Insulators », IEEE Transactions On Dielectrics and Electrical Insulation, vol.6, 1999, pp. 605-611.

Llovera P., Molinié P., «New methodology for surface potential decay measurements applications to the study of charge injection dynamics on polypropylene films », IEEE Transactions On Dielectrics and Electrical Insulation, vol.11, 2004, pp 1049-1056.

Molinié P., «Measuring and Modeling Transient Insulator Response to Charging: the Contribution of Surface Potential Studies» IEEE Transactions On Dielectrics and Electrical Insulation, vol.12, 2005, pp. 939-950.

Moreno V.M., Gorur R.S., «Effect of long term corona on non-ceramic outdoor insulator housing materials », IEEE Transactions On Dielectrics and Electrical Insulation, 2001.

Nguyen D.H., Sylvestre A., Gonon P., Rowe S., «Dielectric properties analysis of silicone rubber », 6th International Conference on Solid Dielectrics, Toulouse, 2004.

Tuncer, E., Gubanski, S.M., «Electrical properties of filled silicone rubber », Journal of Physics: Condensed Matter, Vol.12, 2000, pp. 1873-1897.

Yoshimura N., Kumagai Nishimura S., «Electrical and Environmental Aging of Silicone Rubber used in outdoor insulation », IEEE Transactions on Dielectrics and Electrical Insulation, vol. 6, 1999, pp.632-647. 